\title{
Monochiral helimagnetism in homochiral crystals of $\mathrm{CsCuCl}_{3}$
}

\author{
Y. Kousaka, ${ }^{1,2}$ T. Koyama, ${ }^{3}$ K. Ohishi, ${ }^{4}$ K. Kakurai, ${ }^{4}$ V. Hutanu,${ }^{5}$ H. Ohsumi,${ }^{6}$ T. Arima, ${ }^{7}$ A. Tokuda, ${ }^{8}$ M. Suzuki, ${ }^{9}$ \\ N. Kawamura, ${ }^{9}$ A. Nakao, ${ }^{4}$ T. Hanashima, ${ }^{4}$ J. Suzuki, ${ }^{4}$ J. Campo, ${ }^{10}$ Y. Miyamoto, ${ }^{3}$ A. Sera, ${ }^{3}$ K. Inoue, ${ }^{2,3}$ and J. Akimitsu ${ }^{1,2}$ \\ ${ }^{1}$ Research Institute for Interdisciplinary Science, Okayama University, Okayama, Okayama 700-8530, Japan \\ ${ }^{2}$ Center for Chiral Science, Hiroshima University, Higashi-hiroshima, Hiroshima 739-8526, Japan \\ ${ }^{3}$ Graduate School of Science, Hiroshima University, Higashi-hiroshima, Hiroshima 739-8526, Japan \\ ${ }^{4}$ Neutron Science and Technology Center, Comprehensive Research Organization for Science and Society (CROSS), \\ Tokai, Ibaraki 319-1106, Japan \\ ${ }^{5}$ Institute of Crystallography, RWTH Aachen University and Jülich Centre for Neutron Science (JCNS) at Heinz, Maier-Leibnitz Zentrum \\ (MLZ), 85747 Garching, Germany \\ ${ }^{6}$ RIKEN SPring-8 Center, Sayo, Hyogo 679-5148, Japan \\ ${ }^{7}$ Department of Advanced Materials Science, University of Tokyo Kashiwa, Chiba 277-8561, Japan \\ ${ }^{8}$ Department of Physics, Kwansei Gakuin University, Sanda, Hyogo 669-1337, Japan \\ ${ }^{9}$ Japan Synchrotron Radiation Research Institute, Sayo, Hyogo 679-5198, Japan \\ ${ }^{10}$ Aragón Materials Science Institute (CSIC-University of Zaragoza), 50009 Zaragoza, Spain
}

(Received 6 August 2017; published 12 December 2017)

\begin{abstract}
We report a crystal growth method to obtain homochiral single crystals of $\mathrm{CsCuCl}_{3}$ and polarized neutron diffraction studies to examine the chiral helimagnetism of this compound. The homochiral crystals were grown by two-step crystallization. First, millimeter-sized seed crystals were synthesized by spontaneous crystallization with stirring. The handedness of the seed crystals was determined by x-ray diffraction. Then, centimeter-sized homochiral crystals were obtained from the selected homochiral seed crystals. The large homochiral crystals made it possible to perform polarized neutron diffraction. We clarified a strong correlation between the crystal and magnetic chiralities, which governs the nature of antisymmetric Dzyaloshinskii-Moriya interactions.
\end{abstract}

DOI: 10.1103/PhysRevMaterials.1.071402

The chirality concept, meaning right or left handedness, plays a very important role in various research fields from basic to applied sciences. Regarding new materials from theoretical and experimental sides, it is crucial to understand how to control the chirality in molecules, crystals, and magnetic structures. Chiral helimagnetic structures, forming only one-handed screw magnetic structures, have attracted attention because of the emergence of unique topological magnetic textures such as chiral skyrmion lattices [1] and chiral magnetic soliton lattices [2,3]. The former texture corresponds to a periodic array of magnetic vortices with a fixed swirling direction, while the latter is a periodic array of helical twists partitioned by ferromagnetic regions. Therefore, it is very important to investigate the interplay between crystallographic and helimagnetic chirality because the sense of the rotation of the chiral helimagnetic structures strongly depends on the chiral crystal structure which allows an antisymmetric Dzyaloshinskii-Moriya (DM) interaction caused by the relativistic spin-orbit interaction $[4,5]$.

However, there have been few experimental works on the relationship between crystallographic and magnetic chirality because of the difficulty in controlling the crystallographic chirality. B20-type transition-metal monosilicides are one of the exceptional magnetic compounds where crystals with a particular handedness are obtained. MnSi forms only a left-handed (LH) chiral crystal structure and LH chiral helimagnetic structure [6,7]. However, the series of compounds $\mathrm{Fe}_{1-x} \mathrm{Co}_{x} \mathrm{Si}$ for $x \leqslant 0.15$ form only LH crystalline structures and the opposite, i.e., right-handed ( $\mathrm{RH})$, magnetic structures. The crystalline chirality switches to RH in $x \geqslant 0.20$, and the magnetic chirality also switches to $\mathrm{LH}$ [8]. In this respect, the correlation between the crystalline and magnetic chirality is still controversial in $B 20$ compounds, and it is necessary to investigate it in other chiral magnetic compounds. However, most of the chiral inorganic compounds form racemic-twinned crystals, having both RH and LH crystalline domains. An exceptional crystal is the water-soluble chiral compound $\mathrm{NaClO}_{3}$ for which the use of conventional spontaneous crystallization techniques forms racemic-twinned crystals. However, by stirring the solution during the crystallization process, homochiral crystals can be obtained [9].

As illustrated in Fig. 1, $\mathrm{CsCuCl}_{3}$ crystallizes in the $\mathrm{RH}$ $\left(P 6_{1} 22\right)$ and $\mathrm{LH}\left(P 6_{5} 22\right)$ chiral space groups [10-13]. Unpolarized neutron diffraction studies showed helimagnetic ordering with the magnetic propagation vector $k_{\text {mag }}=(1 / 3,1 / 3, \delta)$ ( $\delta \sim 0.09$ ) below $10.5 \mathrm{~K}$ [14], suggesting a chiral screw structure with a period of $210 \AA$.

We have grown single crystals of $\mathrm{CsCuCl}_{3}$ and probed the handedness of the crystals by means of circularly polarized resonant $\mathrm{x}$-ray diffraction $[15,16]$. We found that millimeter-sized single crystals, obtained by a conventional aqueous solution technique, always formed racemic-twinned domains, which consist of tens $\mu \mathrm{m}$ grains with RH and LH chiralities [15]. Some crystals of several hundreds micrometers sometimes exhibit a homochiral domain [16].

Plakhty et al. performed polarized neutron diffraction experiments in large single crystals of $\mathrm{CsCuCl}_{3}$, consisting of almost one crystal domain of $\mathrm{RH}$, and evaluating the domain ratio between $\mathrm{RH}$ and $\mathrm{LH}$ helimagnetic ordering. They found that the ratio was nearly the same [17], claiming that the Dzyaloshinskii axial vector does not influence the helix chiral degeneracy. This result was inconsistent with the strong 


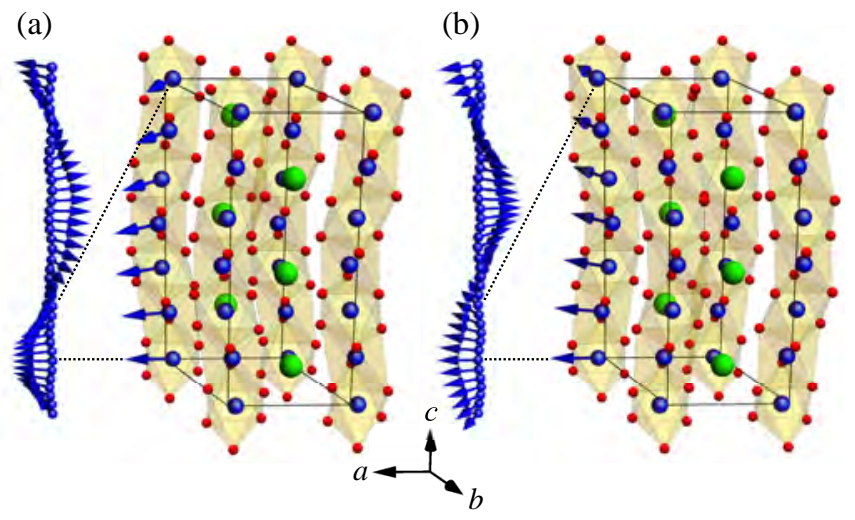

FIG. 1. Crystal structures of (a) right-handed (space group $P 6_{1} 22$ ) and (b) left-handed (space group $\left.P 6_{5} 22\right) \mathrm{CsCuCl}_{3}$. Large, medium, and small balls represent $\mathrm{Cs}, \mathrm{Cu}$, and $\mathrm{Cl}$ ions, respectively. The arrows in a $\mathrm{Cu}$ chain shown in (a) and (b) represent right-handed and left-handed helimagnetic structures, respectively.

correlation between crystal and magnetic chirality observed in transition-metal monosilicides. Our studies [15,16] also indicated that the previous polarized neutron diffraction studies most probably were performed in racemic-twinned crystals [17].

In this Rapid Communication, we demonstrate that large homochiral crystals can be grown by means of spontaneous crystallization with stirring and we evaluate their crystal chirality by using circularly polarized x-ray diffraction. The obtained large homochiral single crystals enabled us to perform polarized neutron diffraction experiments in order to determine the ratio between $\mathrm{RH}$ and $\mathrm{LH}$ magnetic helices, and to clarify the interplay between crystalline and magnetic chirality.

Single crystals of racemic-twinned $\mathrm{CsCuCl}_{3}$ were prepared from a conventional aqueous solution containing $\mathrm{CsCl}$ and $\mathrm{CuCl}_{2}$ by slow evaporation of the slightly acidified solvent. The content of $\mathrm{CuCl}_{2}$ in the solution was increased to $10 \%$ above the stoichiometric composition to avoid the undesired formation of $\mathrm{Cs}_{2} \mathrm{CuCl}_{4}$ [14-16].

To obtain homochiral single crystals of $\mathrm{CsCuCl}_{3}$, the solution was constantly stirred in a beaker during the crystallization process. Details of the crystal growth conditions are described elsewhere [18]. The obtained crystals have a needle shape with a maximum size of $1 \mathrm{~mm}$ diameter and $3 \mathrm{~mm}$ length along the $c$ axis.

X-ray absolute structure analysis using anomalous scattering was performed to evaluate the crystallographic chirality. By comparing the intensities of hundreds of Bragg reflections between $(h, k, l)$ and $(\bar{h}, \bar{k}, \bar{l})$, known as Bijvoet pairs [19], the deducted Flack parameter [20,21] shows the ratio of $\mathrm{RH}$ to LH crystal structures in the domains. By evaluating Flack parameters obtained by x-ray oscillation photographs, we found that the obtained crystals are homochiral crystals, at least at the surface level.

In order to examine the chirality inside the crystal, we picked up a sample with a RH homochiral surface. Then, the sample was cut at the center, and cleaved to explore the $(0,0,1)$ surface. To determine the chiral crystalline population on the cleaved surface, resonant circularly polarized x-ray microdiffraction experiments were performed at the BL39XU in SPring-8, Japan. The incident photon energy was set to be $8.996 \mathrm{keV}$ in the vicinity of the $\mathrm{Cu} K$ edge with a focused $\mathrm{x}$ ray down to $2.7 \times 2.7 \mu \mathrm{m}^{2}$, and a $(0,0,14)$ screw axis forbidden reflection was observed.

To evaluate the difference in intensities between RH circular polarization (RCP) and LH circular polarization (LCP), the chiral crystalline flipping ratio $R_{\chi \text { cryst }}$ is defined as

$$
R_{\chi \text { cryst }}=\frac{I_{\mathrm{RCP}}-I_{\mathrm{LCP}}}{I_{\mathrm{RCP}}+I_{\mathrm{LCP}}},
$$

where $I_{\mathrm{RCP}}$ and $I_{\mathrm{LCP}}$ are the intensities of RCP and LCP, respectively. For the $(0,0,14)$ reflection, the chiral crystalline flipping ratio is described as

$$
R_{\text {x cryst }}=-\frac{2 \sin \theta}{1+\sin ^{2} \theta}\left[\Phi\left(P 6_{1} 22\right)-\Phi\left(P 6_{5} 22\right)\right],
$$

where $\Phi\left(P 6_{1} 22\right)$ and $\Phi\left(P 6_{5} 22\right)$ are volume fractions of $\mathrm{RH}$ and LH chiral crystalline domains, respectively.

Figure 2 shows an image of the crystalline chirality domain on the cleaved $(0,0,1)$ surface. Due to the flatness of the cleaved surface, we can evaluate the flipping ratio for determining the chiral crystal domains in the area surrounded by the dotted lines, having an intensity of $(0,0,14)$ with a signalto-background ratio $>1$, where the signal and background represent the intensity of RCP at on-peak-top and off-peak-top $\omega$ angles, respectively.

As shown in Fig. 2(b), the examined area exhibits a negative value of $R_{\chi \text { cryst }}$, indicating the $\mathrm{RH}$ chiral crystalline domain. The result means that the single crystals obtained by our crystallization technique with stirring are homochiral crystals. In the case of a conventional crystallization process without stirring, the obtained crystals distribute a micrometer mixture of RH and LH chiral crystal domains [15]. This is because many microcrystalline nuclei combine into one racemic-twinned crystal. However, in the case of crystal growth with stirring, a millimeter-sized homochiral single crystal is obtained from only one crystal nucleus. The water flow deprives the growing nucleus of opportunities to be merged with another nucleus in the crystallization process.

To grow homochiral single crystals that are large enough to perform polarized neutron diffraction studies, we performed a second process with the use of a homochiral crystal, described above, as a seed crystal. Spontaneous crystallization started with only one seed crystal in the saturated solution. As the crystallization proceeded, many new pieces of crystals were generated in the solution. They were carefully removed to prevent them from combining with the growing seed crystal. After crystal growth for several weeks, the crystal size became $5 \mathrm{~mm}$ diameter and 10-15 $\mathrm{mm}$ length. To evaluate the crystallographic chirality of the centimeter-sized crystalline sample, we cut small portions at the opposite edges of the sample, and examined them by means of an x-ray oscillation photograph. The crystal chirality of all the pieces was the same as that of the seed crystal.

The crystal structure at $4 \mathrm{~K}$ was determined after collecting and refining, by using JANA2006 [22], 1959 nuclear reflections at the single-crystal neutron diffractometer BL18 (SENJU) at the Materials and Life Science Facility (MLF) of J- 


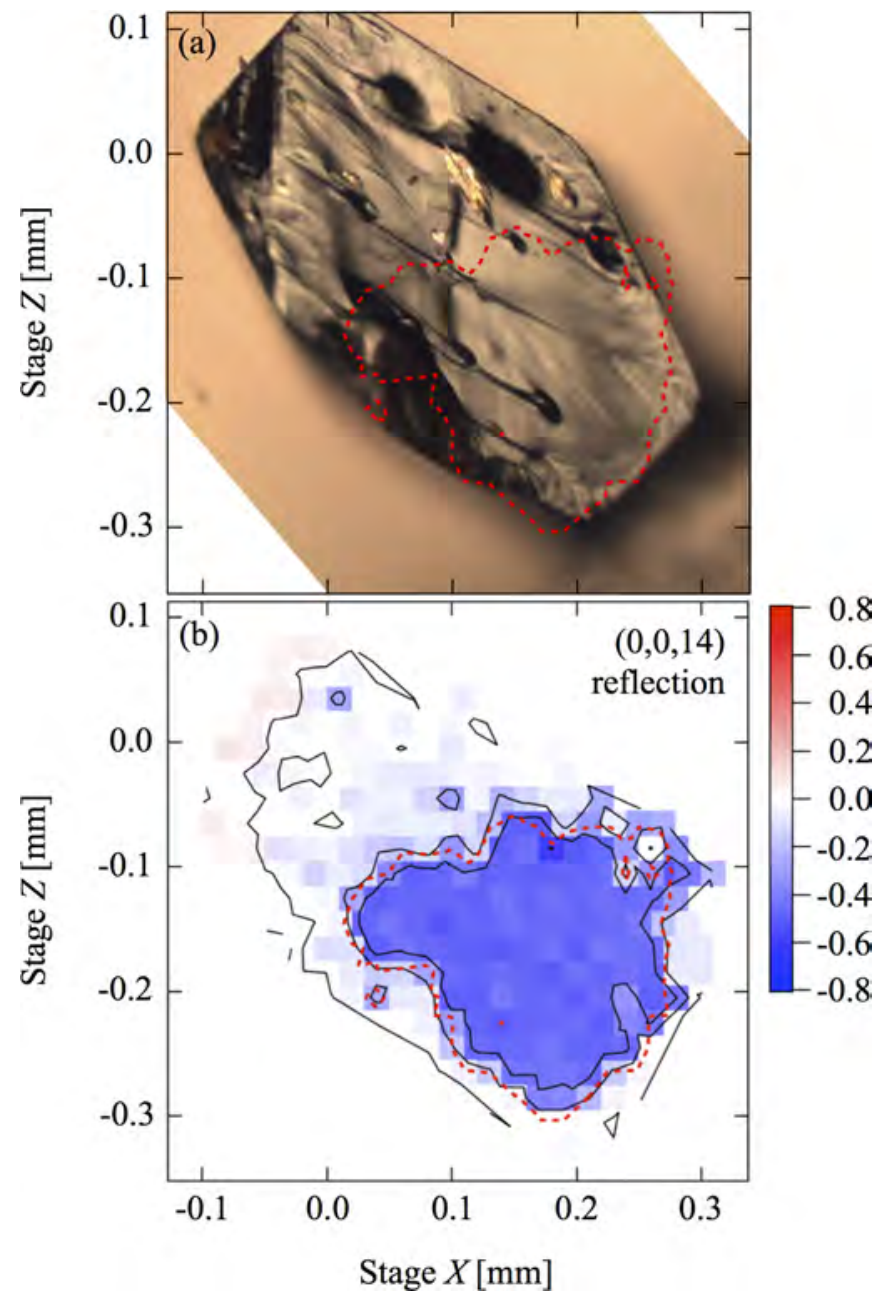

FIG. 2. Imaging of crystalline chirality domains of $\mathrm{CsCuCl}_{3}$ obtained by spontaneous crystallization with stirring. (a) Photograph of the cleaved $(0,0,1)$ surface of a single-crystalline sample projected from the direct synchrotron $\mathrm{x}$-ray beam, and (b) flipping ratio distribution of the $(0,0,14)$ screw axis forbidden reflection. Areas inside the red dotted lines have a $(0,0,14)$ diffraction intensity with a signal-to-background ratio $>1$. The scale is denoted by the color bar. The blue and red areas represent the right-handed $P 6_{1} 22$ and the left-handed $P 6_{5} 22$ domains, respectively.

PARC (Japan). The refined structure with reliable parameters $R F_{\text {obs }}=0.0511$ and $w R F_{\text {obs }}=0.0881$ was well consistent with that of the x-ray oscillation photograph.

The observed and calculated nuclear structure factors for the $(1,1, l)$ reflections are shown in Table I. It is clear that for unpolarized neutron diffraction there is no difference between the calculated nuclear structure factors for the $\mathrm{RH}$ $\left(P 6_{1} 22\right)$ and LH $\left(P 6_{5} 22\right)$ symmetries, making it impossible to distinguish the handedness of the crystal structure. Our calculation and experiments do not reproduce the tendency observed in Refs. [14,17].

The polarized neutron diffraction technique is known as a very powerful method to detect the domain ratio between $\mathrm{RH}$ and LH screw magnetic structures due to the chiral term of the magnetic scattering, expressed as [23,24]

$$
M(\mathbf{Q})_{\chi}^{2}=-i\left[\mathbf{M}(\mathbf{Q})_{\perp} \times \mathbf{M}(\mathbf{Q})_{\perp}^{*}\right]_{x} .
$$

TABLE I. Observed and calculated nuclear structure factors of $(1,1, l)$ and its equivalent reflections, based on space groups of righthanded $P 6_{1} 22$ and left-handed $P 6_{5} 22$.

\begin{tabular}{lcrr}
\hline \hline & & \multicolumn{2}{c}{ Calculation } \\
\cline { 3 - 4 } Reflection & Observation & $P 6_{1} 22$ & $P 6_{5} 22$ \\
\hline$(1,1,1)$ & $13.34(14)$ & 11.22 & 11.22 \\
$(1,1,2)$ & $3.36(8)$ & 3.07 & 3.07 \\
$(\overline{1}, 1,3)$ & $8.89(11)$ & 10.21 & 10.21 \\
$(\overline{1}, \overline{1}, \overline{4})$ & $183.64(52)$ & 170.60 & 170.60 \\
$(1,1,5)$ & $53.88(54)$ & 59.78 & 59.78 \\
$(\overline{1}, \overline{1}, 6)$ & $82.99(83)$ & 88.11 & 88.11 \\
$(\overline{1}, \overline{1}, 7)$ & $76.14(76)$ & 80.17 & 80.17 \\
$(\overline{2}, 1,8)$ & $30.75(33)$ & 31.68 & 31.68 \\
$(\overline{1}, \overline{1}, 9)$ & $24.75(26)$ & 23.64 & 23.64 \\
$(\overline{1}, \overline{1}, 10)$ & $57.86(59)$ & 57.65 & 57.65 \\
$(\overline{1}, \overline{1}, 11)$ & $11.73(23)$ & 6.82 & 6.82 \\
$(\overline{1}, \overline{1}, 12)$ & $74.45(76)$ & 75.32 & 75.32 \\
\hline \hline
\end{tabular}

Here, $x$ is one of three orthogonal directions and parallel to the scattering vector $Q . \mathbf{M}(\mathbf{Q})_{\perp}$ is the magnetic interaction vector. The spherical neutron polarimetry technique effectively accesses the chiral term [25-27]. We note that the neutron spin polarization of the incoming and the scattered beam is analyzed in the three orthogonal directions. $I_{i, f}$ is the scatter intensity, where $i$ and $f$ represent the incoming and outgoing neutron polarization, respectively. The chiral term can be directly detected by measuring spin-flip (SF) cross sections of $I_{+x,-x}$ and $I_{-x,+x}$, expressed as

$$
I_{ \pm x, \mp x}=\left|\mathbf{M}(\mathbf{Q})_{\perp}\right|^{2} \mp i\left[\mathbf{M}(\mathbf{Q})_{\perp} \times \mathbf{M}(\mathbf{Q})_{\perp}^{*}\right]_{x} .
$$

To evaluate the difference between $I_{+x,-x}$ and $I_{-x,+x}$, the chiral magnetic flipping ratio $R_{\chi \text { mag }}$ is defined as

$$
R_{\chi \mathrm{mag}}=\frac{I_{+x,-x}-I_{-x,+x}}{I_{+x,-x}+I_{-x,+x}} .
$$

Therefore, the chiral magnetic flipping ratio is described as

$$
R_{\chi \mathrm{mag}}=\frac{-i\left[\mathbf{M}(\mathbf{Q})_{\perp} \times \mathbf{M}(\mathbf{Q})_{\perp}^{*}\right]_{x}}{\left|\mathbf{M}(\mathbf{Q})_{\perp}\right|^{2}}
$$

Polarized neutron diffraction experiments were performed on the BL15 (TAIKAN) in the MLF, and on instrument POLI at Maier-Leibnitz Zentrum (MLZ) in Germany [28]. RH and LH homochiral single crystals were prepared for the experiments. A single-crystal spherical neutron polarimetry technique using CRYOPAD was carried out at the POLI diffractometer operated with a two-axis mode with a wavelength $\lambda=0.89 \AA$. Neutron polarization and analysis were achieved by ${ }^{3} \mathrm{He}$-spin filters in the incoming and scattered beams [29-32]. The incident and final neutron spin polarizations are chosen in three orthogonal directions $x, y$, and $z$, where $x$ is defined to be parallel to the scattering vector, $y$ to be perpendicular to $x$ and the $[\overline{1}, 2,0]^{*}$ axis, and $z$ to be parallel to the $[\overline{1}, 2,0]^{*}$ axis.

The magnetic satellite peaks were observed with a $\omega$ scan at $4 \mathrm{~K}$, and incomplete neutron polarization due to ${ }^{3} \mathrm{He}$-spin filters was safely corrected by using a transmission monitor for the incoming beam and nuclear Bragg reflections at $(0,0,12)$ for the scattered beam, as described elsewhere 

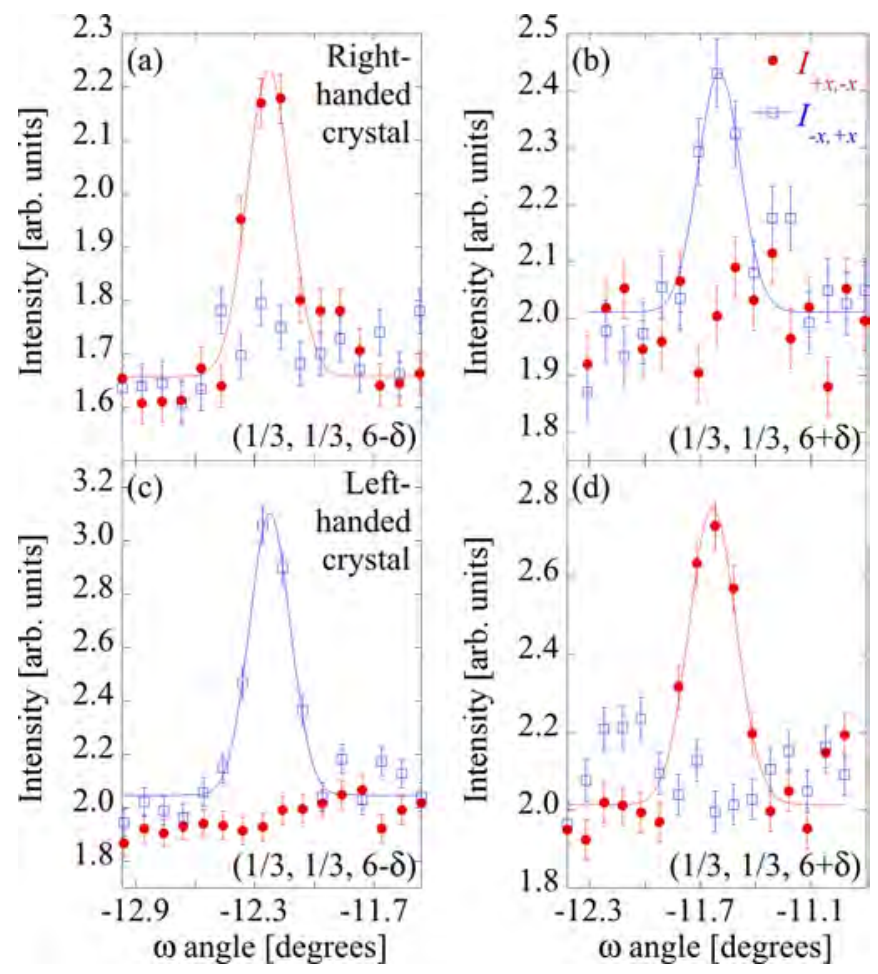

FIG. 3. Spin-flip channel $\omega$-scan profiles of (a) $(1 / 3,1 / 3,6-\delta)$ and (b) $(1 / 3,1 / 3,6+\delta)$ magnetic Bragg reflections for a right-handed $\left(P 6_{1} 22\right)$ homochiral crystal and of (c) $(1 / 3,1 / 3,6-\delta)$ and (d) $(1 / 3,1 / 3,6+\delta)$ for a left-handed $\left(P 6_{5} 22\right)$ homochiral crystal. Solid circles and open squares represent the measured intensities, and continuous lines are Gaussian fits to the data.

[31]. Measuring SF cross sections $+x,-x$ and $-x,+x$ of the magnetic satellite reflections, e.g., $(1 / 3,1 / 3,6-\delta)$ and $(1 / 3,1 / 3,6+\delta)$, respectively, gives direct insight about the magnetic chirality of the sample. From the relative intensities of those two channels, the ratio between RH and LH magnetic domains can be determined. The rocking curves of the SF channels of the magnetic satellites are depicted in Fig. 3 for RH and LH crystals. For the $(1 / 3,1 / 3,6-\delta)$ reflection in the RH crystal, shown in Fig. 3(a), we observed the magnetic satellite peak only when the neutron polarization was along $+x,-x$. However, for the $(1 / 3,1 / 3,6+\delta)$ reflection, shown in Fig. 3(b), the peak was observed only when the polarization was along $-x,+x$. The opposite behavior happens for the $\mathrm{LH}$ crystal, shown in Figs. 3(c) and 3(d). Table II summarizes the

TABLE II. Observed and calculated chiral magnetic flipping ratios of magnetic satellite reflections in right-handed $\left(P 6_{1} 22\right)$ and left-handed $\left(P 6_{5} 22\right)$ homochiral crystals. $R C h M$ and $L C h M$ represent right- and left-handed chiral magnetic structures, respectively.

\begin{tabular}{crrrrr}
\hline \hline \multirow{2}{*}{ Reflection } & \multicolumn{2}{c}{ Observation } & & \multicolumn{2}{c}{ Calculation } \\
\cline { 2 - 3 } \cline { 6 - 6 } & \multicolumn{1}{c}{$P 6_{1} 22$} & $P 6_{5} 22$ & & $R C h M$ & LChM \\
\hline$(1 / 3,1 / 3,6-\delta)$ & $0.95(22)$ & $-0.98(17)$ & & 1.0 & -1.0 \\
$(1 / 3,1 / 3,6+\delta)$ & $-0.98(27)$ & $0.91(17)$ & & -1.0 & 1.0 \\
$(2 / 3,2 / 3,6+\delta)$ & $-1.03(15)$ & $0.95(21)$ & & -1.0 & 1.0 \\
\hline \hline
\end{tabular}

observed and calculated chiral magnetic flipping ratios in each magnetic satellite peak. The observed chiral magnetic flipping ratios indicate that the $\mathrm{RH}$ crystalline $\mathrm{CsCuCl}_{3}$ forms a purely RH helimagnetic structure, and the LH crystal forms a purely LH helimagnetic structure, as shown by the arrows in Fig. 1.

We successfully determined the correlation between crystalline and helimagnetic chirality by using homochiral single crystals and polarized neutron diffraction measurements. Earlier, Plakhty et al. [17] reported that in a quantum antiferromagnet with a triangular lattice $\mathrm{CsCuCl}_{3}$, that belongs to the $X Y$ chiral universality class [33], the handedness of the helical magnetic structure was not directly coupled with structural chirality. Their misinterpretation might be caused by the difficulties in growing homochiral single crystals and in determining the crystal chirality by use of neutron diffraction.

To determine the crystallographic chirality, anomalous scattering of the $\mathrm{x}$ ray, containing real and imaginary parts, is necessary. On the other hand, neutron diffraction experiments of $\mathrm{CsCuCl}_{3}$ cannot distinguish $\mathrm{RH}$ and $\mathrm{LH}$ chiral crystal structures because the nuclear scattering lengths of $\mathrm{Cs}, \mathrm{Cu}$, and $\mathrm{Cl}$ have no imaginary parts.

Taking advantage of our spontaneous crystallization with stirring for producing homochiral seed crystals in combination with a careful examination of crystallographic chirality using conventional and circularly polarized x-ray, followed by the laborious growth of enantiopure specimens, produces centimeter-sized homochiral single crystals of $\mathrm{CsCuCl}_{3}$ with the desired handedness. This method may be generally applied for producing samples to study a wide variety of chiral physical phenomena, such as, e.g., optical activity, magnetochiral dichroism (MCD), nonreciprocal spin-wave propagation, etc. [34,35].

Using spherical neutron polarimetry could be shown that in a prototypical triangular lattice antiferromagnet $\mathrm{CsCuCl}_{3}$, the handedness of the magnetic helicity is coupled and directly controlled by the crystallographic lattice chirality. These results could be understood in terms of a DM interaction strongly coupled to the lattice. The Dzyaloshinskii axial vector removes the helix chiral degeneracy, leaving only one magnetic helix with handedness dependent on the sample crystal chirality.

The present study will enhance the fundamental aspects of the DM interaction in terms of microscopic mechanisms, together with studies on intermetallic chiral magnetic compounds such as monoaxial ferromagnetic chiral crystals $\mathrm{CrNb}_{3} \mathrm{~S}_{6}$ and $B 20$ transition-metal monosilicides.

We express our sincere appreciation to Y. Togawa and J. Kishine for fruitful discussions. This work was supported by JSPS KAKENHI Grants No. 25220803, No. 25390139, No. 26108719, No. 15H03680, No. 15H05885, No. 15H05886, No. 16KK0102, 17H02912, No. 17H02767, and No. 17H02815. K.K. acknowledges the support by the Helmholtz International Fellow Award. J.C. acknowledges Grant No. MAT2015-68200-C2-2-P from the Spanish Ministry of Economy and Competitiveness. The synchrotron radiation experiments were performed at the BL39XU of SPring- 8 with the approval of the Japan Synchrotron Radiation Research Institute (JASRI) (Proposals No. 2009A1607 and No. 2009B1586). The neutron experiments at the MLF of the J-PARC were 
performed under user programs (Proposals No. 2013A0166, No. 2013B0108, No. 2014A0085, and No. 2014B0165). Part of the work is based upon experiments performed at the POLI instrument, which is operated by RWTH Aachen University and Forschungszentrum Jülich GmbH (Jülich Aachen Research Alliance JARA).
[1] S. Muhlbauer, B. Binz, F. Jonietz, C. Pfleiderer, A. Rosch, A. Neubauer, R. Georgii, and P. Böni, Science 323, 915 (2009).

[2] Y. Togawa, T. Koyama, K. Takayanagi, S. Mori, Y. Kousaka, J. Akimitsu, S. Nishihara, K. Inoue, A. S. Ovchinnikov, and J. Kishine, Phys. Rev. Lett. 108, 107202 (2012).

[3] Y. Togawa, Y. Kousaka, K. Inoue, and J. Kishine, J. Phys. Soc. Jpn. 85, 112001 (2016).

[4] I. Dzyaloshinsky, J. Phys. Chem. Solids 4, 241 (1958).

[5] T. Moriya, Phys. Rev. 120, 91 (1960).

[6] M. Tanaka, H. Takayoshi, M. Ishida, and Y. Endoh, J. Phys. Soc. Jpn. 54, 2970 (1985).

[7] M. Ishida, Y. Endoh, S. Mitsuda, Y. Ishikawa, and M. Tanaka, J. Phys. Soc. Jpn. 54, 2975 (1985).

[8] S. V. Grigoriev, D. Chernyshov, V. A. Dyadkin, V. Dmitriev, S. V. Maleyev, E. V. Moskvin, D. Menzel, J. Schoenes, and H. Eckerlebe, Phys. Rev. Lett. 102, 037204 (2009).

[9] D. K. Kondepudi, R. J. Kaufman, and N. Singh, Science 250, 975 (1990).

[10] A. W. Schlueter, R. A. Jacobson, and R. E. Rundle, Inorg. Chem. 5, 277 (1966).

[11] C. J. Kroese, J. C. M. Tindemans-van Eyndhoven, and W. J. A. Masskant, Solid State Commun. 9, 1707 (1971).

[12] C. J. Kroese, W. J. A. Masskant, and G. C. Verschoor, Acta Crystallogr., Sect. B 30, 1053 (1974).

[13] S. Hirotsu, J. Phys. C 10, 967 (1977).

[14] K. Adachi, N. Achiwa, and M. Mekata, J. Phys. Soc. Jpn. 49, 545 (1980)

[15] H. Ohsumi, A. Tokuda, S. Takeshita, M. Takata, M. Suzuki, N. Kawamura, Y. Kousaka, J. Akimitsu, and T. Arima, Angew. Chem., Int. Ed. 52, 8718 (2013).

[16] Y. Kousaka, H. Ohsumi, T. Komesu, T. Arima, M. Takata, S. Sakai, M. Akita, K. Inoue, T. Yokobori, Y. Nakao, E. Kaya, and J. Akimitsu, J. Phys. Soc. Jpn. 78, 123601 (2009).

[17] V. Plakhty, J. Wosnitza, J. Kulda, T. Bruckel, W. Schweika, D. Visser, S. Gavrilov, E. Moskvin, R. Kremer, and M. Banks, Physica B 385-386, 288 (2006).
[18] Y. Kousaka, T. Koyama, M. Miyagawa, K. Tanaka, J. Akimitsu, and K. Inoue, J. Phys.: Conf. Ser. 502, 012019 (2014).

[19] J. M. Bijvoet, Nature (London) 173, 888 (1954).

[20] H. D. Flack, Acta Crystallogr., Sect. A 39, 876 (1983).

[21] G. Berardinelli and H. D. Flack, Acta Crystallogr., Sect. A 41, 500 (1985).

[22] V. Petricek, M. Dusek, and L. Palatinus, Jana2006: The Crystallographic Computing System (Institute of Physics, Praha, 2006).

[23] M. Blume, Phys. Rev. 130, 1670 (1963).

[24] K. Siratori, J. Akimitsu, E. Kita, and M. Nishi, J. Phys. Soc. Jpn. 48, 1111 (1980).

[25] T. Finger, D. Senff, K. Schmalzl, W. Schmidt, L. P. Regnault, P. Becker, L. Bohaty, and M. Braden, Phys. Rev. B 81, 054430 (2010).

[26] M. Baum, J. Leist, T. Finger, K. Schmalzl, A. Hiess, L. P. Regnault, P. Becker, L. Bohaty, G. Eckold, and M. Braden, Phys. Rev. B 89, 144406 (2014).

[27] J. Stein, M. Baum, S. Holbein, T. Cronert, V. Hutanu, A. C. Komarek, and M. Braden, J. Phys.: Condens. Matter 27, 446001 (2015).

[28] V. Hutanu, J. Large-Scale Res. Facil. 1, A16 (2015).

[29] F. Tasset and E. Ressouche, Nucl. Instrum. Methods Phys. Res., Sect. A 359, 537 (1995).

[30] V. Hutanu, M. Meven, E. Lelievre-Berna, and G. Heger, Physica B 404, 2633 (2009).

[31] V. Hutanu, M. Meven, S. Masalovich, G. Heger, and G. Roth, J. Phys.: Conf. Ser. 294, 012012 (2011).

[32] V. Hutanu, W. Luberstetter, E. Bourgeat-Lami, M. Meven, A. Sazonov, A. Steffen, G. Heger, G. Roth, and E. Lelievre-Berna, Rev. Sci. Instrum. 87, 105108 (2016).

[33] H. Kawamura, J. Phys.: Condens. Matter 10, 4707 (1998).

[34] G. L. J. A. Rikken and E. Raupach, Nature (London) 390, 493 (1997).

[35] S. Seki, Y. Okamura, K. Kondou, K. Shibata, M. Kubota, R. Takagi, F. Kagawa, M. Kawasaki, G. Tatara, Y. Otani, and Y. Tokura, Phys. Rev. B 93, 235131 (2016). 